\title{
Study to Standardize the Suitable Protocol for Turf Growing
}

\author{
Madhur Kumar, Devi singh and Bhoopendra Singh* \\ Department of Horticulture, Sam Higginbottom University of Agriculture, Technology and \\ Sciences, Prayagraj, Allahabad, 211007, India \\ *Corresponding author
}

\section{A B S T R A C T}

\begin{tabular}{|l|}
\hline Ke y w o r d s \\
Bermuda grass, \\
Buffalo grass, Turf \\
grass, Nitrogen and \\
grass land
\end{tabular}

\section{Introduction}

Grasslands are among the largest ecosystem on the world representing almost $40 \%$ of the terrestrial area (Reheul et al., 2010). There are three major aspects of grassland's significance; an ecological, economical and an aesthetical aspect. Grassland has a fundamental role in soil and environment protection. It serves as an indispensable source of nutrients and water and harbours over one third of the global stock of carbon in terrestrial ecosystems (Reheul et al., 2010). Top-soil loss and water runoff is much less intensive on grassland compared to arable land (Macleod et al., 2007, 2013). Grassland is also a valuable source of biodiversity, especially in its extensive forms (Gaujour et al., 2012). Grassland species are 
mainly used for forage and amenity purposes. Their rapid establishment and growth form dense swards that deliver highly nutritious, palatable and easily digestible fodder, thereby providing the basis for healthy meat, milk and other animal products used for human consumption.

Turf grasses can be used for i) sports including golf courses and sports fields, ii) landscaping services such as lawns in parks, around homes, schools, institutions and other public areas and iii) functional purposes such as land reclamation on contaminated and derelict industrial sites and low-maintenance ground cover grown on highways and roadside shoulders, airfields and ditches. The aesthetical role of grassland is obvious; attractive landscapes for recreational purposes such as hiking, hunting, camping and photography as well as grazing animals on pastures are part of a multifunctional grassland agriculture that is associated with a healthy lifestyle and deeply anchored in the 21st century society. The associated ecotourism has evolved into a growing market creating new income opportunities for local inhabitants.

Bermuda grasses (Cynodon dactylon) are perhaps the most important and widely adapted warm season grasses in the world. They are thought to have originated in East Africa (Beard, 1973; Emmons, 1995) but are widely distributed throughout tropical and subtropical regions of over 100 countries (Duble, 1996). Throughout the world, Bermuda grass is known by many other names including Couch grass, Devil grass, Week grass, Grimily, Quick grass, and Wiregrass. As some of these names suggest, Bermuda grass is considered a weedy grass in many instances. The genus Cynodon comprises nine species, with $C$. dactylon [L.] Pers., (common Bermuda grass) being the most widespread. Bermuda grass is a vigorous sod-forming perennial that spreads by stolon's and rhizomes, and sometimes seed. It tolerates close mowing, generally between $1.3 \mathrm{~cm}$ and $2.6 \mathrm{~cm}$; however, some dwarf varieties may be mowed at $0.6 \mathrm{~cm}$.

It is adapted to warm humid and semiarid climates with extended periods of high temperatures, mild winters, and moderate to high rainfall (Duble, 1996). Bermuda grass (Cynodon dactylon), a $\mathrm{C}_{4}$ plant, growth and development is interrupted when light interception is reduced. In shade, warm-season turf grass decline is attributed to morphological limitations, such as reduced lateral stem growth (Beard, 1997).

A shaded micro environment initiates excessive shoot vertical growth, depleting turf grass root carbohydrate status (Qian and Engelke, 1999). Previous warm-season and cool-season turf grass evaluations have noted a linear relationship as shade increases, vertical shoot growth increases (Tegg and Lane, 2004).

Soil $\mathrm{pH}$ also affects the activity of soil enzymes through its controls on microbial enzymatic production, ionization-induced conformational changes of enzymes, and/or availability of substrates and enzymatic cofactors. Oxidative enzymes, i.e., phenol oxidase and peroxidase were found to be more sensitive to soil $\mathrm{pH}$ change than the hydrolytic ones and increased with soil pH (Sinsabaugh et al., 2008).

Although turf grasses, including golf courses, parks and home lawns, cover $14 \%$ of the cropland area in the USA and provide both recreational and environmental benefits (Qian and Follett, 2002), there is a widespread concern that turf grasses may not be ecologically sound due to $\mathrm{N}$ loss potentials associated with intensive management.

Frequent mowing is a primary component of turf management. During the active growing season, turf is mowed as often as once a day. 
Mowing produces grass clippings that can filter into the canopy, to the soil surface and decompose. Understanding the dynamics of soil microbial biomass and $\mathrm{N}$ mineralization following clipping addition is central to formulating fertilizer best management practices that minimize $\mathrm{N}$ losses via leaching and de nitrification.

Plant materials consist of a variety of organic compounds, and represent a carbon source for soil microbial metabolism. Incorporation of plant material into the soil generally stimulates microbial growth and activity, while the elemental composition of the material may have more specific effects on $\mathrm{N}$ mineralization and immobilization turnover (MIT).

The magnitude of priming seems to vary with the type of added organic matter. However, there is little information on soil microbial biomass and $\mathrm{N}$ mineralization dynamics following the addition of turf grass clippings. Inherent soil and microbial properties may, to some extent, influence the decomposition of certain plant materials.

However, soil $\mathrm{N}$ availability had little effect on the mineralization of $\mathrm{N}$-rich organic materials such as amino acids (Jones and Shannon, 1999).

Several studies demonstrated that the size and activity of indigenous soil microbial populations affected the decomposition of soil incorporated plant materials (Allison and Killham, 1988; Franzluebbers et al., 1995; Henriksen and Breland, 1999a).

In the present study, we examined short-term soil microbial biomass and $\mathrm{N}$ mineralization dynamics in a turf grass chromo sequence following the addition of turf grass clippings.

\section{Materials and Methods}

The experiment will be carried out at the Central Research Farm, Department of
Horticulture, Sam Higginbottom Institute of Agriculture, Technology \& Science, Allahabad (U.P.) - 211007 during the year 2015-16 and 2016-2017. The experimental farm situated in the river basin of the Ganga and Yamuna. It is situated at $25^{\circ} .57^{\prime} \mathrm{N}$ latitude $81^{\circ} .5^{\prime}$ E longitude with an altitude of $98 \mathrm{~m}$ above the mean sea level.

The soil of the experimental area is sandy loam in texture with soil reaction in almost neutral range $(\mathrm{pH} 7.2)$ organic carbon is $0.51 \%$, nitrogen is available but phosphorus and potash are in medium to higher range. Average annual rainfall is $1100 \mathrm{~mm}$ precipitating mostly in between middle of July to end of September - January is the coldest month when mercury may drop down to an average minimum of $5^{\circ}$ on the other hand May - June are the hottest month recording average high temperature above $46^{\circ} \mathrm{C}$ (Table 1 ).

The experiment was laid out according to split plot design by randomizing varieties in main plots and nitrogen levels in subplots. Equal sized plugs were planted in the plots measuring $1 \mathrm{~m} \mathrm{x} 1 \mathrm{~m}$ in size during $3 \mathrm{rd}$ week of July, 2017 on well prepared and leveled soil surface. Plug to plug distance was kept at 8 inches $(20 \mathrm{~cm})$. Fertilizer was applied according to the treatments; whereas, other management practices like irrigation, weeding and mowing were same for all treatments during entire period of study.

All plots received a weekly application of irrigation water during the growing season. Moreover, turfs were mowed biweekly to a height of $2.0 \mathrm{~cm}$. Grass clippings were removed from the experimental plots. Plugs were allowed to establish for a period of 60 days and then data on different growth and biomass indices of the grasses were collected by adopting standard procedures during the conduct of the experiment. 


\section{Results and Discussion}

Effect of nitrogen variation on the total survival percentage of the bermuda and buffalo grass

Results obtained on total survival percentage of plant depicted that $200 \mathrm{~g} \mathrm{~N} / \mathrm{m}^{2} /$ month proved best treatment as compared to $150 \mathrm{~g}$ $\mathrm{N} / \mathrm{m}^{2} /$ month, $100 \mathrm{~g} \mathrm{~N} / \mathrm{m}^{2} /$ month and $50 \mathrm{~g}$ $\mathrm{N} / \mathrm{m}^{2} /$ month. Whereas, in case of varietal behaviour regarding this parameter, Bermuda grass with Nitrogen @ $200 \mathrm{~g} / \mathrm{m}^{2}$ with moving height $5.0 \mathrm{~mm}$ has shown superiority over Buffalo grass. It was also noted that results were non-significant in case of seasons but the autumn season gives better survival percentage (86.47\%) as compare to other two seasons i.e. Spring season (83.47) and winter season (79.68) (Table 2).

Effect of nitrogen variation on the on death percentage of plant per clipping of the bermuda and buffalo grass

Results obtained on disease and death percentage of plant per clipping depicted that $200 \mathrm{~g} \mathrm{~N} / \mathrm{m}^{2} /$ month proved best treatment as compared to $150 \mathrm{~g} \mathrm{~N} / \mathrm{m}^{2} /$ month, $100 \mathrm{~g}$ $\mathrm{N} / \mathrm{m}^{2} /$ month and $50 \mathrm{~g} \mathrm{~N} / \mathrm{m}^{2} /$ month (Table 3).

Whereas, in case of varietal behaviour regarding this parameter, Bermuda grass with Nitrogen@ $200 \mathrm{~g} / \mathrm{m}^{2}$ with moving height 5.0 $\mathrm{mm}$ has shown superiority over Buffalo grass. It was also noted that results were nonsignificant in case of seasons but the Autumn season gives better disease and death percentage of plant per clipping nodes $(1.23 \%)$ as compare to other two seasons i.e.
Spring season (1.67\%) and winter season $(1.33 \%)$.

Effect of nitrogen variation on the on first sprouting at nodes of the bermuda and buffalo grass

Results obtained on first sprouting at nodes depicted that $200 \mathrm{~g} \mathrm{~N} / \mathrm{m}^{2} /$ month proved best treatment as compared to $150 \mathrm{~g} \mathrm{~N} / \mathrm{m}^{2} /$ month, $100 \mathrm{~g} \mathrm{~N} / \mathrm{m}^{2} /$ month and $50 \mathrm{~g} \mathrm{~N} / \mathrm{m}^{2} /$ month. Whereas, in case of varietal behaviour regarding this parameter, Bermuda grass with Nitrogen@200 g/ $/ \mathrm{m}^{2}$ with moving height 5.0 $\mathrm{mm}$ has shown superiority over Buffalo grass.

It was also noted that results were nonsignificant in case of seasons but the autumn season gives earlier first sprouting at (8.87 days) as compare to other two seasons i.e. Spring season (8.68 days) and winter season (9.00 days) (Table 4$)$.

Effect of nitrogen variation on the on size before every clip of the bermuda and buffalo grass

Results obtained on size before every clip depicted that $200 \mathrm{~g} \mathrm{~N} / \mathrm{m}^{2} /$ month proved best treatment as compared to $150 \mathrm{~g} \mathrm{~N} / \mathrm{m}^{2} /$ month, $100 \mathrm{~g} \mathrm{~N} / \mathrm{m}^{2} /$ month and $50 \mathrm{~g} \mathrm{~N} / \mathrm{m}^{2} /$ month. Whereas, in case of varietal behaviour regarding this parameter, Bermuda grass with Nitrogen@ $200 \mathrm{~g} / \mathrm{m}^{2}$ with moving height 5.0 $\mathrm{mm}$ has shown superiority over Buffalo grass. It was also noted that results were nonsignificant in case of seasons but the Autumn season gives better size before every clip $(10.13 \mathrm{~cm})$ as compare to other two season i.e. Spring season $(8.40 \mathrm{~cm})$ and winter season $(8.07 \mathrm{~cm})$ (Table 5). 
Table.1 Treatments combinations

\begin{tabular}{|c|c|}
\hline T1 & $\mathrm{N}_{1} \mathrm{M}_{1} \mathrm{G}_{1}$ - Bermuda grass with Nitrogen @ $50 \mathrm{~g} / \mathrm{m}^{2}$ with moving height $3.0 \mathrm{~mm}$ \\
\hline $\mathbf{T 2}$ & $\mathrm{N}_{1} \mathrm{M}_{2} \mathrm{G}_{1}$ - Bermuda grass with Nitrogen @ $50 \mathrm{~g} / \mathrm{m}^{2}$ with moving height $4.0 \mathrm{~mm}$ \\
\hline T3 & $\mathrm{N}_{1} \mathrm{M}_{3} \mathrm{G}_{1}$ - Bermuda grass with Nitrogen @ $50 \mathrm{~g} / \mathrm{m}^{2}$ with moving height $5.0 \mathrm{~mm}$ \\
\hline T4 & $\mathrm{N}_{2} \mathrm{M}_{1} \mathrm{G}_{1}$ - Bermuda grass with Nitrogen @ $100 \mathrm{~g} / \mathrm{m}^{2}$ with moving height $3.0 \mathrm{~mm}$ \\
\hline T5 & $\mathrm{N}_{2} \mathrm{M}_{2} \mathrm{G}_{1}$ - Bermuda grass with Nitrogen @ $100 \mathrm{~g} / \mathrm{m}^{2}$ with moving height $4.0 \mathrm{~mm}$ \\
\hline T6 & $\mathrm{N}_{2} \mathrm{M}_{3} \mathrm{G}_{1}$ - Bermuda grass with Nitrogen @ $100 \mathrm{~g} / \mathrm{m}^{2}$ with movi \\
\hline T7 & $\mathrm{N}_{3} \mathrm{M}_{1} \mathrm{G}_{1}$ - Bermuda grass with Nitrogen @ $150 \mathrm{~g} / \mathrm{m}^{2}$ with moving height $3.0 \mathrm{~mm}$ \\
\hline T8 & $\mathrm{N}_{3} \mathrm{M}_{2} \mathrm{G}_{1}$ - Bermuda grass with Nitrogen @ $150 \mathrm{~g} / \mathrm{m}^{2} \mathrm{w}$ \\
\hline T9 & $\mathrm{N}_{3} \mathrm{M}_{3} \mathrm{G}_{1}$ - Bermuda grass with Nitrogen @ $150 \mathrm{~g} / \mathrm{m}^{2}$ with moving height $5.0 \mathrm{~mm}$ \\
\hline T10 & $\mathrm{N}_{4} \mathrm{M}_{1} \mathrm{G}_{1}$ - Bermuda grass with Nitrogen @ $200 \mathrm{~g} / \mathrm{m}^{2}$ with moving height $3.0 \mathrm{~mm}$ \\
\hline T11 & $\mathrm{N}_{4} \mathrm{M}_{2} \mathrm{G}_{1}$ - Bermuda grass with Nitrogen @ $200 \mathrm{~g} / \mathrm{m}^{2}$ with moving height $4.0 \mathrm{~mm}$ \\
\hline T12 & $\mathrm{N}_{4} \mathrm{M}_{3} \mathrm{G}_{1}$ - Bermuda grass with Nitrogen @ $200 \mathrm{~g} / \mathrm{m}^{2}$ with moving height $5.0 \mathrm{~mm}$ \\
\hline T13 & $\mathrm{N}_{1} \mathrm{M}_{1} \mathrm{G}_{1}$ - Buffalograss \\
\hline T14 & $\mathrm{N}_{1} \mathrm{M}_{2} \mathrm{G}_{1}$ - Buffalograss with Nitrogen @ $50 \mathrm{~g} / \mathrm{m}^{2}$ with moving height $4.0 \mathrm{~mm}$ \\
\hline T15 & $\mathrm{N}_{1} \mathrm{M}_{3} \mathrm{G}_{1}$ - Buffalograss with Nitrogen @ $50 \mathrm{~g} / \mathrm{m}^{2}$ with moving height $5.0 \mathrm{~mm}$ \\
\hline T16 & $\mathrm{N}_{2} \mathrm{M}_{1} \mathrm{G}_{1}$ - Buffalograss with Nitrogen @ $100 \mathrm{~g} / \mathrm{m}^{2}$ with moving height $3.0 \mathrm{~mm}$ \\
\hline T17 & $\mathrm{N}_{2} \mathrm{M}_{2} \mathrm{G}_{1}$ - Buffalograss with Nitrogen @ $100 \mathrm{~g} / \mathrm{m}^{2}$ with moving height $4.0 \mathrm{~mm}$ \\
\hline T18 & $\mathrm{N}_{2} \mathrm{M}_{3} \mathrm{G}_{1}$ - Buffalograss with Nitrogen @ $100 \mathrm{~g} / \mathrm{m}^{2}$ with moving height $5.0 \mathrm{~mm}$ \\
\hline T19 & $\mathrm{N}_{3} \mathrm{M}_{1} \mathrm{G}_{1}$ - Buffalograss with Nitrogen @ $150 \mathrm{~g} / \mathrm{m}^{2}$ with moving height $3.0 \mathrm{~mm}$ \\
\hline $\mathbf{T 2 0}$ & $\mathrm{N}_{3} \mathrm{M}_{2} \mathrm{G}_{1}$ - Buffalograss with Nitrogen @ $150 \mathrm{~g} / \mathrm{m}^{2}$ with moving height $4.0 \mathrm{~mm}$ \\
\hline T21 & $\mathrm{N}_{3} \mathrm{M}_{3} \mathrm{G}_{1}$ - Buffalograss with Nitrogen @ $150 \mathrm{~g} / \mathrm{m}^{2}$ with moving height $5.0 \mathrm{~mm}$ \\
\hline T22 & $\mathrm{N}_{4} \mathrm{M}_{1} \mathrm{G}_{1}$ - Buffalograss with Nitrogen @ $200 \mathrm{~g} / \mathrm{m}^{2}$ with moving height $3.0 \mathrm{~mm}$ \\
\hline $\mathbf{T 2 3}$ & $\mathrm{N}_{4} \mathrm{M}_{2} \mathrm{G}_{1}$ - Buffalograss with Nitrogen @ $200 \mathrm{~g} / \mathrm{m}^{2}$ with moving height $4.0 \mathrm{~mm}$ \\
\hline T24 & $\mathrm{N}_{4} \mathrm{M}_{3} \mathrm{G}_{1}$ - Buffalograss with Nitrogen @ $200 \mathrm{~g} / \mathrm{m}^{2}$ with moving height $5.0 \mathrm{~mm}$ \\
\hline
\end{tabular}


Table.2 Total Survival percentage

\begin{tabular}{|c|c|c|c|c|}
\hline Treatment & $\begin{array}{c}\text { AUTUMN } \\
\text { SEASON } \\
(\%)\end{array}$ & $\begin{array}{c}\text { SPRING } \\
\text { SEASON } \\
(\%)\end{array}$ & $\begin{array}{c}\text { WINTER } \\
\text { SEASON } \\
(\%)\end{array}$ & Pooled \\
\hline T1 & 72.78 & 77.56 & 70.77 & 73.70 \\
\hline T2 & 72.88 & 76.56 & 70.48 & 73.31 \\
\hline T3 & 73.77 & 71.65 & 71.88 & 72.43 \\
\hline T4 & 74.88 & 74.65 & 71.98 & 73.84 \\
\hline T5 & 75.38 & 77.55 & 72.98 & 75.30 \\
\hline T6 & 76.37 & 76.87 & 73.98 & 75.74 \\
\hline T7 & 78.47 & 76.65 & 74.13 & 76.42 \\
\hline T8 & 80.57 & 75.65 & 74.79 & 77.00 \\
\hline T9 & 77.64 & 79.54 & 75.32 & 77.50 \\
\hline T10 & 84.26 & 82.75 & 76.75 & 81.25 \\
\hline T11 & 84.97 & 80.93 & 78.74 & 81.55 \\
\hline T12 & 86.47 & 83.47 & 79.68 & 83.21 \\
\hline T13 & 74.48 & 75.27 & 64.87 & 71.54 \\
\hline T14 & 74.37 & 75.65 & 64.99 & 71.67 \\
\hline T15 & 73.95 & 74.65 & 65.75 & 71.45 \\
\hline T16 & 75.37 & 73.29 & 66.69 & 71.78 \\
\hline T17 & 76.36 & 75.60 & 67.34 & 73.10 \\
\hline T18 & 74.35 & 76.60 & 68.13 & 73.03 \\
\hline T19 & 79.37 & 76.59 & 68.90 & 74.95 \\
\hline T20 & 81.37 & 75.65 & 70.77 & 75.93 \\
\hline T21 & 78.54 & 79.75 & 70.48 & 76.26 \\
\hline T22 & 81.92 & 81.85 & 71.88 & 78.55 \\
\hline T23 & 82.46 & 82.66 & 71.98 & 79.03 \\
\hline T24 & 83.37 & 83.88 & 72.98 & 80.08 \\
\hline CD value & $\mathbf{8 . 3 6}$ & $\mathbf{8 . 1 6}$ & $\mathbf{8 . 2 3}$ & $\mathbf{8 . 4 5}$ \\
\hline F Value & $\mathbf{1 1 . 7 6 5 * *}$ & $\mathbf{1 0 . 9 7 6} * *$ & $\mathbf{1 1 . 2 3 1} * *$ & $\mathbf{1 1 . 1 2 5 * *}$ \\
\hline & & & & \\
\hline
\end{tabular}


Int.J.Curr.Microbiol.App.Sci (2019) 8(12): 2767-2779

Table.3 Death percentage of plant per clipping

\begin{tabular}{|c|c|c|c|c|}
\hline Treatment & $\begin{array}{c}\text { AUTUMN } \\
\text { SEASON }\end{array}$ & $\begin{array}{c}\text { SPRINGR } \\
\text { SEASON }\end{array}$ & $\begin{array}{c}\text { WINTER } \\
\text { SEASON }\end{array}$ & Pooled \\
\hline T1 & 4.67 & 5.67 & 5.33 & 5.22 \\
\hline T2 & 3.33 & 4.33 & 4.33 & 4.00 \\
\hline T3 & 4.67 & 5.67 & 4.33 & 4.89 \\
\hline T4 & 3.00 & 4.67 & 4.33 & 4.00 \\
\hline T5 & 3.33 & 4.00 & 4.00 & 3.78 \\
\hline T6 & 4.00 & 4.67 & 4.33 & 4.33 \\
\hline T7 & 3.00 & 3.67 & 3.33 & 3.33 \\
\hline T8 & 3.00 & 2.67 & 2.33 & 2.67 \\
\hline T9 & 1.33 & 2.33 & 2.00 & 1.89 \\
\hline T10 & 4.00 & 3.33 & 3.00 & 3.44 \\
\hline T11 & 2.67 & 2.67 & 2.33 & 2.56 \\
\hline T12 & 1.23 & 1.67 & 1.33 & 1.41 \\
\hline T13 & 2.67 & 7.00 & 6.67 & 5.44 \\
\hline T14 & 1.33 & 6.33 & 6.00 & 4.56 \\
\hline T15 & 3.00 & 6.33 & 6.00 & 5.11 \\
\hline T16 & 4.67 & 5.67 & 5.33 & 5.22 \\
\hline T17 & 2.33 & 4.67 & 4.33 & 3.78 \\
\hline T18 & 3.00 & 4.33 & 4.00 & 3.78 \\
\hline T19 & 1.33 & 4.00 & 3.67 & 3.00 \\
\hline T20 & 2.00 & 4.00 & 3.67 & 3.22 \\
\hline T21 & 1.67 & 3.67 & 3.33 & 2.89 \\
\hline T22 & 1.33 & 3.33 & 3.00 & 2.56 \\
\hline T23 & 1.33 & 3.00 & 2.67 & 2.33 \\
\hline T24 & 1.12 & 2.33 & 2.00 & 1.82 \\
\hline CD value & 4.56 & 4.25 & 4.12 & $\mathbf{4 . 2 5}$ \\
\hline F Value & $\mathbf{3 . 4 5 3}$ & $\mathbf{3 . 6 0 8}$ & $\mathbf{3 . 2 5 5}$ & $\mathbf{3 . 4 3 6}$ \\
\hline & & & & \\
\hline
\end{tabular}


Table.4 First sprouting at nodes (days)

\begin{tabular}{|c|c|c|c|c|}
\hline Treatment & $\begin{array}{c}\text { AUTUMN } \\
\text { SEASON }\end{array}$ & $\begin{array}{l}\text { SPRINGR } \\
\text { SEASON }\end{array}$ & $\begin{array}{l}\text { WINTER } \\
\text { SEASON }\end{array}$ & POOLED \\
\hline T1 & 13.33 & 13.33 & 14.67 & 13.78 \\
\hline $\mathbf{T} 2$ & 12.00 & 12.00 & 13.67 & 12.56 \\
\hline T3 & 9.33 & 9.33 & 13.33 & 10.67 \\
\hline T4 & 12.67 & 12.67 & 13.00 & 12.78 \\
\hline T5 & 11.00 & 11.00 & 13.00 & 11.67 \\
\hline T6 & 12.00 & 12.00 & 12.67 & 12.22 \\
\hline T7 & 11.00 & 11.00 & 11.33 & 11.11 \\
\hline T8 & 11.33 & 11.33 & 11.67 & 11.44 \\
\hline T9 & 10.33 & 10.33 & 10.67 & 10.44 \\
\hline T10 & 10.00 & 10.00 & 10.33 & 10.11 \\
\hline T11 & 9.33 & 9.33 & 9.67 & 9.44 \\
\hline T12 & 8.67 & 8.67 & 9.00 & 8.78 \\
\hline T13 & 15.00 & 15.00 & 16.67 & 15.56 \\
\hline T14 & 15.33 & 15.33 & 16.33 & 15.67 \\
\hline T15 & 13.00 & 13.00 & 15.33 & 13.78 \\
\hline T16 & 14.00 & 14.00 & 14.67 & 14.22 \\
\hline T17 & 13.67 & 13.67 & 14.33 & 13.89 \\
\hline T18 & 12.67 & 12.67 & 13.33 & 12.89 \\
\hline T19 & 12.33 & 12.33 & 13.33 & 12.67 \\
\hline T20 & 13.00 & 13.00 & 13.00 & 13.00 \\
\hline T21 & 11.67 & 11.67 & 12.33 & 11.89 \\
\hline T22 & 11.00 & 11.00 & 11.33 & 11.11 \\
\hline T23 & 11.33 & 11.33 & 11.67 & 11.44 \\
\hline T24 & 10.67 & 10.67 & 11.00 & 10.78 \\
\hline CD value & 4.78 & 4.85 & 4.48 & 4.56 \\
\hline F Value & $7.385 * *$ & $6.698 * *$ & $7.242 * *$ & $7.347 * *$ \\
\hline
\end{tabular}


Table.5 Size before every clips (cm)

\begin{tabular}{|c|c|c|c|c|}
\hline Treatment & $\begin{array}{l}\text { AUTUMN } \\
\text { SEASON }\end{array}$ & $\begin{array}{l}\text { SPRINGR } \\
\text { SEASON }\end{array}$ & $\begin{array}{l}\text { WINTER } \\
\text { SEASON }\end{array}$ & POOLED \\
\hline T1 & 6.30 & 5.83 & 5.50 & 5.88 \\
\hline $\mathbf{T} 2$ & 6.47 & 5.93 & 5.60 & 6.00 \\
\hline T3 & 6.80 & 6.17 & 5.83 & 6.27 \\
\hline $\mathbf{T 4}$ & 7.17 & 6.43 & 6.10 & 6.57 \\
\hline T5 & 7.53 & 6.70 & 6.37 & 6.87 \\
\hline T6 & 7.80 & 6.83 & 6.50 & 7.04 \\
\hline T7 & 8.20 & 7.10 & 6.77 & 7.36 \\
\hline T8 & 8.50 & 7.30 & 6.97 & 7.59 \\
\hline T9 & 9.10 & 7.77 & 7.43 & 8.10 \\
\hline T10 & 9.30 & 7.83 & 7.50 & 8.21 \\
\hline T11 & 9.80 & 8.20 & 7.87 & 8.62 \\
\hline T12 & 10.13 & 8.40 & 8.07 & 8.87 \\
\hline T13 & 4.63 & 4.73 & 4.40 & 4.59 \\
\hline T14 & 4.67 & 4.73 & 4.40 & 4.60 \\
\hline T15 & 4.87 & 4.90 & 4.57 & 4.78 \\
\hline T16 & 5.10 & 5.10 & 4.77 & 4.99 \\
\hline T17 & 5.13 & 5.10 & 4.77 & 5.00 \\
\hline T18 & 5.17 & 5.13 & 4.80 & 5.03 \\
\hline T19 & 5.10 & 5.03 & 4.70 & 4.94 \\
\hline T20 & 5.17 & 5.13 & 4.80 & 5.03 \\
\hline T21 & 5.30 & 5.17 & 4.83 & 5.10 \\
\hline T22 & 5.30 & 5.20 & 4.87 & 5.12 \\
\hline T23 & 5.53 & 5.33 & 5.00 & 5.29 \\
\hline T24 & 5.63 & 5.37 & 5.03 & 5.34 \\
\hline CD value & 3.86 & 3.25 & 3.29 & 3.57 \\
\hline F Value & $9.453 * *$ & $9.115 * *$ & $9.210 * *$ & $9.342 * *$ \\
\hline
\end{tabular}


Table.6 Size of clipped grass after cutting

\begin{tabular}{|c|c|c|c|c|}
\hline Treatment & $\begin{array}{l}\text { AUTUMN } \\
\text { SEASON }\end{array}$ & $\begin{array}{l}\text { SPRINGR } \\
\text { SEASON }\end{array}$ & $\begin{array}{l}\text { WINTER } \\
\text { SEASON }\end{array}$ & POOLED \\
\hline T1 & 4.30 & 3.83 & 3.50 & 3.88 \\
\hline $\mathbf{T} 2$ & 4.47 & 3.93 & 3.60 & 4.00 \\
\hline T3 & 4.80 & 4.17 & 3.83 & 4.27 \\
\hline T4 & 5.17 & 4.43 & 4.10 & 4.57 \\
\hline T5 & 5.53 & 4.70 & 4.37 & 4.87 \\
\hline T6 & 5.80 & 4.83 & 4.50 & 5.04 \\
\hline T7 & 6.20 & 5.10 & 4.77 & 5.36 \\
\hline T8 & 6.50 & 5.30 & 4.97 & 5.59 \\
\hline T9 & 7.10 & 5.77 & 5.43 & 6.10 \\
\hline T10 & 7.30 & 5.83 & 5.50 & 6.21 \\
\hline T11 & 7.80 & 6.20 & 5.87 & 6.62 \\
\hline T12 & 8.13 & 6.40 & 6.07 & 6.87 \\
\hline T13 & 2.63 & 2.73 & 2.40 & 2.59 \\
\hline T14 & 2.67 & 2.73 & 2.40 & 2.60 \\
\hline T15 & 2.87 & 2.90 & 2.57 & 2.78 \\
\hline T16 & 3.10 & 3.10 & 2.77 & 2.99 \\
\hline T17 & 3.13 & 3.10 & 2.77 & 3.00 \\
\hline T18 & 3.17 & 3.13 & 2.80 & 3.03 \\
\hline T19 & 3.10 & 3.03 & 2.70 & 2.94 \\
\hline T20 & 3.17 & 3.13 & 2.80 & 3.03 \\
\hline T21 & 3.30 & 3.17 & 2.83 & 3.10 \\
\hline T22 & 3.30 & 3.20 & 2.87 & 3.12 \\
\hline T23 & 3.53 & 3.33 & 3.00 & 3.29 \\
\hline T24 & 3.63 & 3.37 & 3.03 & 3.34 \\
\hline CD value & 2.31 & 3.12 & 2.71 & 2.46 \\
\hline F Value & $4.356 * *$ & $4.125 * *$ & 4.110*** & $4.243 *$ \\
\hline
\end{tabular}


Table.7 Number of stolen

\begin{tabular}{|c|c|c|c|c|}
\hline Treatment & $\begin{array}{c}\text { AUTUMN } \\
\text { SEASON }\end{array}$ & $\begin{array}{c}\text { SPRINGR } \\
\text { SEASON }\end{array}$ & $\begin{array}{c}\text { WINTER } \\
\text { SEASON }\end{array}$ & POOLED \\
\hline T1 & 9.00 & 8.30 & 7.70 & 8.33 \\
\hline T2 & 9.30 & 8.70 & 8.00 & 8.67 \\
\hline T3 & 9.70 & 9.00 & 8.30 & 9.00 \\
\hline T4 & 8.70 & 8.00 & 7.30 & 8.00 \\
\hline T5 & 10.00 & 9.30 & 8.70 & 9.33 \\
\hline T6 & 10.70 & 10.00 & 9.30 & 10.00 \\
\hline T7 & 10.70 & 10.00 & 9.30 & 10.00 \\
\hline T8 & 11.70 & 11.00 & 10.30 & 11.00 \\
\hline T9 & 11.00 & 10.30 & 9.70 & 10.33 \\
\hline T10 & 12.30 & 11.70 & 11.00 & 11.67 \\
\hline T11 & 12.00 & 11.30 & 10.70 & 11.33 \\
\hline T12 & 13.00 & 12.30 & 11.70 & 12.33 \\
\hline T13 & 6.70 & 6.00 & 5.30 & 6.00 \\
\hline T14 & 7.00 & 6.30 & 5.70 & 6.33 \\
\hline T15 & 7.30 & 6.70 & 6.00 & 6.67 \\
\hline T16 & 8.30 & 7.70 & 7.00 & 7.67 \\
\hline T17 & 9.00 & 8.30 & 7.70 & 8.33 \\
\hline T18 & 9.70 & 9.00 & 8.30 & 9.00 \\
\hline T19 & 9.70 & 9.00 & 8.30 & 9.00 \\
\hline T20 & 11.00 & 10.30 & 9.70 & 10.33 \\
\hline T21 & 11.30 & 10.70 & 10.00 & 10.67 \\
\hline T22 & 11.30 & 10.70 & 10.00 & 10.67 \\
\hline T23 & 12.00 & 11.30 & 10.70 & 11.33 \\
\hline T24 & 13.00 & 12.30 & 11.70 & 12.33 \\
\hline CD value & $\mathbf{6 . 2 6}$ & $\mathbf{5 . 8 7}$ & $\mathbf{5 . 6 8}$ & $\mathbf{5 . 8 6}$ \\
\hline F Value & $\mathbf{7 . 5 4 7 * *}$ & $\mathbf{6 . 4 7 3 * *}$ & $\mathbf{6 . 1 4 2} * *$ & $\mathbf{6 . 4 5 8 * *}$ \\
\hline & & & & \\
\hline
\end{tabular}

Effect of nitrogen variation on the on size of clipped grass after cutting of the bermuda and buffalo grass

Results obtained on Size of clipped grass after cutting depicted that $200 \mathrm{~g} \mathrm{~N} / \mathrm{m}^{2} /$ month proved best treatment as compared to $150 \mathrm{~g}$ $\mathrm{N} / \mathrm{m}^{2} /$ month, $100 \mathrm{~g} \mathrm{~N} / \mathrm{m}^{2} /$ month and $50 \mathrm{~g}$ $\mathrm{N} / \mathrm{m}^{2} /$ month. Whereas, in case of varietal behaviour regarding this parameter, Bermuda grass with Nitrogen@ $200 \mathrm{~g} / \mathrm{m}^{2}$ with moving height $5.0 \mathrm{~mm}$ has shown superiority over Buffalo grass. It was also noted that results were non-significant in case of seasons but the Autumn season gives better Size of clipped grass after cutting $(8.13 \mathrm{~cm})$ as compare to other two season i.e. Spring season $(6.40 \mathrm{~cm})$ and winter season $(6.07 \mathrm{cnm})$ (Table 6).

Effect of nitrogen variation on the on number of stolen of the bermuda and buffalo grass

Results obtained on Number of stolen depicted that $200 \mathrm{~g} \mathrm{~N} / \mathrm{m}^{2} / \mathrm{month}$ proved best treatment as compared to $150 \mathrm{~g} \mathrm{~N} / \mathrm{m}^{2} /$ month, $100 \mathrm{~g}$ 
$\mathrm{N} / \mathrm{m}^{2} / \mathrm{month}$ and $50 \mathrm{~g} \mathrm{~N} / \mathrm{m}^{2} / \mathrm{month}$. Whereas, in case of varietal behaviour regarding this parameter, Bermuda grass with Nitrogen@ $200 \mathrm{~g} / \mathrm{m}^{2}$ with moving height $5.0 \mathrm{~mm}$ has shown superiority over Buffalo grass. It was also noted that results were non-significant in case of seasons but the autumn season gives better Number of stolen (13.00) as compare to other two seasons i.e. spring season (12.30) and winter season (11.70) (Table 7).

The role of $\mathrm{N}$ fertilization in enhancing the production of aboveground matter in bermuda grasses is widely known (Overman et al., 1990; Sartain and Dudeck, 1982; Snyder and Cisar, 2000; Stanford et al., 2005; Trenholm et al., 1998). In this study, $\mathrm{N}$ rate influenced aerial dry weight, the number of primary stolons, and their ramifications, but not their length or their number of nodes. On the contrary, other authors found an increasing effect of $\mathrm{N}$ on stolon length of 'Tifdwarf' (Stanford et al., 2005; Trenholm et al., 1997). The number of primary stolons was negatively affected by the absence of $\mathrm{N}$ fertilization (zero $\mathrm{N}$ rate), while no differences were observed among the other $\mathrm{N}$ rates despite a significant nonlinear regression The number of secondary stolons increased along with $\mathrm{N}$ increase fitting a linear regression model, but only the difference between zero $\mathrm{N}$ and triple $\mathrm{N}$ was significant.

Nitrogen application improved different growth and biomass indices of turf grasses vigorously with $200 \mathrm{~g} \mathrm{~N} / \mathrm{m}^{2} /$ month application presenting superior results as compared to rest of the treatments for almost all parameters of study. Moreover, Bermuda grass responded better to $\mathrm{N}$ application than Buffalo grass whereas seasonal variations were negligible with slight superiority of autumn season over spring in some cases. Therefore, optimum use of nitrogen fertilizer in small split doses would help better towards vigorous growth of turf grasses and to keep your lawns and green spaces attractive and healthy.

\section{References}

Beard, J.B. (1997). Shade stresses and adaptation mechanisms of turf grasses. Int. Turf grass Soc. Res. J. 8:11861196.

Farrar K, Bryant DN, Turner L, Gallagher JA, Thomas A, Farrell M, et al., (2012) Breeding for bio-ethanol production in Lilium perenne L.: association of allelic variation with high watersoluble carbohydrate content. Bio energy Res ;5:149-57.

Gaujour E, Amiaud B, Mignolet C, Plantureux S. (2012) Factors and processes affecting plant biodiversity in permanent grasslands. A review. Agron Sustainable Dev;32:133-60.

Macleod CJA, Binley A, Hawkins SL, Humphreys MW, Turner LB, Whalley WR, et al.,(2007) Genetically modified hydrographs: what can grass genetics do for temperate catchment hydrology Hydrol Process, 21: 2217-21.

Macleod CJA, Humphreys MW, Whalley WR, Turner L, Binley A, Watts CW, et al.,(2013) A novel grass hybrid to reduce flood generation in temperate regions. Sci Rep., 3: 1683.

Qian, Y.L. and M.C. Engelke. (1999). Influence of trinexapac-ethyl on diamond zoysia grass in a shaded environment. Crop Sci. 39:202-208.

Reheul D, De Cauwer B, Cougnon M. (2010). The role of forage crops in multifunctional agriculture. In: Boller B, Posselt UK, Veronesi F, editors. Fodder crops and amenity grasses, handbook of plant breeding. New York: Springer; p. 1-12.

Roshni Agnihotri, S.L. Chawla1 and Vivek Kumar (2015) Use of plant growth regulators in turf grasses: A review IN: Bioved, 26(2): 367-376.

Shiping Chen, Yongfei Bai, Lixia Zhang, Xingguo Han (2005).Comparing 
physiological responses of two Tegg, R.S. and P. A. Lane. (2004). A dominant grass species to nitrogen comparison of the performance and addition in Xilin River Basin of China.IN: Environmental and growth of a range of turf grass species Experimental Botany 53: 65-75. under shade. Australian Journal of Experimental Agriculture.44:353- 358

\section{How to cite this article:}

Madhur Kumar, Devi singh and Bhoopendra Singh. 2019. Study to Standardize the Suitable Protocol for Turf Growing. Int.J.Curr.Microbiol.App.Sci. 8(12): 2767-2779. doi: https://doi.org/10.20546/ijcmas.2019.812.324 